\title{
EMOTION ANALYSIS USING SELF-TRAINING ON MALAYSIAN CODE-MIXED TWITTER DATA
}

\author{
Kathleen Swee Neo Tan, Tong Ming Lim and Yee Mei Lim \\ Department of Computer Science and Embedded Systems, Faculty of Computing and Information Technology, \\ Tunku Abdul Rahman University College, Kuala Lumpur, Malaysia
}

\begin{abstract}
Microblogs such as Twitter has made available a vast resource of User Generated Content (UGC) on which emotion analysis may be performed. Organizations increasingly value the opinions obtained from emotion analysis. These insights help to drive decision-making activities and provide constructive inputs to engage their customers and services. In a multiracial country such as Malaysia, it is common to find that tweets are written in mixed languages of Malay, Malaysian slang and English. These tweets increase the complexity of the emotion analysis task, especially considering that there is a serious lack of labeled data available in order to make use of supervised learning techniques. This paper explores the use of self-training, a semi-supervised technique that only requires a small initial labeled dataset to conduct emotion analysis of Malaysian code-mixed Twitter data. The results are promising as the accuracy achieved is higher compared to the baseline models.
\end{abstract}

\section{KEYWORDS}

Emotion Analysis, Code-Mixed, Twitter, Self-Training, Sentiment Analysis

\section{INTRODUCTION}

There has been an increasing use of microblogs. People express their sentiments towards a certain object, be it a product, an organization, a person, an event or a policy on microblogs. Various organizations have adopted microblogs as a means of branding, marketing and maintaining customer relations. Among the microblog platforms, Facebook and Twitter are the most popular microblog sites in Malaysia (Statcounter, 2020) and have emerged as platforms that enable not only rapid dissemination of information but also has the ability to influence other people's sentiments on a topic that was written about.

Another development that has been observed recently is the growing interest in the area of sentiment and emotion analysis on microblog text. The aim of sentiment analysis is to identify positive and negative polarity expressed in the text. Emotion analysis, on the other hand, identifies the specific emotion (such as joy, sadness or anger) that is being expressed in the text. Organizations such as commercial companies, governments, political parties and non-governmental organizations (NGOs) realize that there is a vast amount of data available from which they can obtain useful insights regarding the sentiments and emotions of their customers, citizens and people in general. This allows organizations to arrive at better decisions and timely actions.

Malaysians are generally able to speak in at least two different languages due to the multi-racial composition of its society. This unique characteristic has made it very natural for Malaysians to speak and write using mixed languages. For example, it is quite common to tweet using a mix of Malay and English, a phenomenon known as code-mixing. An example of a code-mixed tweet is "Stress tu adalah apabila barang dah sold out and kau kene marah dengan customers???? Hmmmmmm murung hari aku", which means "Stress is when your products are sold out and you're scolded by your customers???? Hmmmmmm my day is sad". These code-mixed tweets impose a number of challenges when trying to perform emotion analysis. Firstly, the language used is a mix of formal as well as informal single-word terms and Multi-Word Expressions (MWE). Secondly, non-standard sentence structures are used due to the fact that the constituent languages of the code-mixed text have different vocabulary and grammatical characteristics (Lo et al., 2016). Thirdly, there are limited available labeled code-mixed datasets to serve as training datasets (Aghababaei and 
Makrehchi, 2017). Fourthly, there is a lack of sentiment and emotion resources for languages other than English and even more so for code-mixed languages (Dashtipour et al., 2016).

In view of the above-mentioned problems, semi-supervised learning has become a viable technique to be utilized (Hemmatian and Sohrabi, 2019). Although its performance is usually not as good as supervised learning techniques, it has the important benefit that it does not require a large labeled dataset for training (Da Silva et al., 2016). This paper explores the use of self-training, a semi-supervised technique that only requires a small initial labeled dataset, to conduct emotion analysis of Malaysian code-mixed Twitter data. Specifically, experiments to evaluate the accuracy of self-training with different confidence thresholds and $\mathrm{n}$-gram features are carried out on Malaysia Twitter data. In the experiments conducted, emotion analysis is treated as a multi-label classification problem with six categories which correspond to the basic emotions happy, sadness, love, fear, anger and surprise.

\section{RELATED WORK}

He and Zhou (2011) proposed a self-training approach that used labeled features to classify positive and negative sentiments. The labeled features contained words with prior sentiment orientation obtained from the MPQA subjectivity lexicon (Wilson et al., 2005) . The lexicon was used to build an initial classifier using generalized expectation (GE) criteria (Mann and McCallum, 2010), to perform predictions on an unlabeled dataset. The samples labeled with high confidence were then collected to form a labeled dataset. Their framework was evaluated on the movie review dataset (Pang and Lee, 2005), which comprised 1000 positive and 1000 negative movie reviews, and a multi-domain sentiment dataset (Blitzer et al., 2007). Their results showed a better accuracy $(75.10 \%)$ compared to the baseline model. However, this approach does not address multi-label classification and is difficult to implement for languages which do not have available sentiment lexicons.

Haimovitch et al. (2012) used AROW (Crammer et al., 2013), an online algorithm based on confidence weighted learning, to build a sentiment analysis model. Their model was evaluated on datasets comprising Amazon product reviews from 8 domains that were labeled as positive or negative based on the number of stars accompanying their review. From each dataset, 1000 examples were randomly selected to form the initial dataset. They reported that for all domains, the test error was $40 \%$ lower compared to the test error of the baseline. They also found that since AROW is an online algorithm, the order and rate of using the data affects the performance of the final classifier. As the metrics used in their paper was test error, it is not possible to compare against the performance in other work which typically uses either accuracy or F1-score.

Han et al. (2019) proposed a self-training model that comprised two stages. First, the model utilized dynamic thresholds for the confidence scores, with higher threshold values for the initial iterations and lower threshold values for the later iterations to cater for the declining confidence scores of the predicted labels in later iterations. Next, multiple Support Vector Machine (SVM) classifiers based on the bagging algorithm were used in order to avoid overfitting and reduce the impact of misclassification errors. A weighted voting strategy was then applied to combine the results from the multiple classifiers and determine the final prediction. They evaluated their model using different initial labeled training dataset sizes ranging from 500 to 1000 and reported that their proposed model achieved F1-scores ranging between $79.96 \%$ and $82.69 \%$, outperforming the baseline methods. The higher F1-scores were achieved when the larger initial labeled datasets were used.

The self-training model built by Iosifidis and Ntoutsi (2019) used Multinomial Naïve Bayes (MNB) as the base learner with unigrams as features to classify tweets as positive, negative or neutral. They experimented with different confidence thresholds in the range 65-100\% with 5 iterations and reported that the accuracy obtained for the iterations was lower than that of the initial model, which is an expected outcome as the training set is progressively expanded through predictions. The final accuracy achieved in their experiments ranged between approximately $89.5 \%$ and $93.5 \%$. They concluded that tweets are far different from comments or reviews as the character limitations may cause the structure of a tweet to vary significantly compared to other texts. In addition, the different spanning periods may result in changes in the words and their relevance to different classes. 
Most of the previous work used self-training to classify text into positive and negative sentiments (Da Silva et al., 2016) and there is very limited work that utilized the self-training approach for multi-label emotion classification on code-mixed text.

\section{EXPERIMENTAL DESIGN}

In this paper, emotion analysis was treated as a multi-label classification problem with six categories which correspond to the basic emotions: happy, sadness, love, fear, anger and surprise.

Self-training is a wrapper-based semi-supervised approach (Da Silva et al., 2016) whereby a supervised learning classifier is used as a base learner which is "wrapped" within an algorithm which iteratively (1) trains a classifier using a small labeled dataset, (2) carries out prediction on a much larger unlabeled dataset, and (3) transfers the predicted examples with high confidence scores from the unlabeled dataset to the labeled dataset. The confidence score for a given example is considered high if its value exceeds a set threshold value. These three steps are repeated either for a pre-determined number of iterations or until the unlabeled dataset is exhausted.

In this study, SVM was used as the base learner. The feature set used was Term Frequency-Inverse Document Frequency (TF-IDF) and n-grams. TF-IDF is a statistical score that indicates the importance of a term in a dataset. It is obtained by multiplying the normalized frequency of the term in the tweet with the $\log$-scaled inverse frequency of the term in the entire dataset. For the initial labeled dataset, it was ensured that there was a balanced representation of each emotion. Experiments were conducted to investigate the effect of using different confidence thresholds and the various n-gram features.

The dataset used for the experiments was the publicly available Malaya emotion dataset (Husein, 2018) which is a collection of Twitter data organized into six files according to the emotions happy, sadness, love, fear, anger and surprise. The tweets in this dataset are mostly in Malay and Malaysian slang, as well as code-mixed Malay-English text. A few examples of the tweets in the dataset with their labels are provided in Table 1. Details of the dataset's composition according to the different emotion classes are shown in Table 2.

Table 1. Samples of tweets in the Malaya emotion dataset

\begin{tabular}{lc}
\hline Sample tweet & Emotion label \\
\hline Awek cantik, high maintenance. Takut kita & fear \\
Why is it internet like siput babi when I want to do something important & anger \\
ohh itu your mommy, not mine dah kenapa dia voice note? rindu barang kali & love \\
at least aku siap 2 assigment dah lega weh & happy \\
Same here Allahu sedih but life goes on & sadness \\
Sy tlmpau sakit hati tengok muka dia nai... hahaha like im speechless tkjut sis & surprise \\
\hline
\end{tabular}

Table 2. Malaya emotion dataset composition by emotion

\begin{tabular}{cc}
\hline Emotion label & Number of tweets \\
\hline fear & 18,895 \\
anger & 51,745 \\
love & 59,242 \\
happy & 48,924 \\
sadness & 79,233 \\
surprise & 37,778 \\
\hline Total tweets & 295,817 \\
\hline
\end{tabular}

In order to evaluate the overall performance of self-training on the code-mixed Twitter data, accuracy was use as the evaluation metric. Additionally, F1-score was used to compare the performance of self-training in terms of the individual emotion labels. The baseline model used in this study was the traditional SVM trained on the initial labeled dataset of 600 examples alone as the training set. 


\section{EXPERIMENTS}

\subsection{Data Preprocessing}

Basic text preprocessing was carried out and this included the removal of tokens such as stopwords, mentions (@), URLs, emails and telephone numbers, as these tokens do not reflect any emotion. The stopword list was built by merging the English stopwords obtained from nltk with the Malay stopword list published by The Proteus Project (2004). In addition, the words were converted to lowercase.

As can be observed in Table 2, the subset of tweets categorized with the emotion fear had the lowest number $(18,895)$ of tweets. To avoid bias due to imbalanced representation of classes in the training dataset, a balanced dataset was created by first randomly extracting 18,895 tweets from each emotion subset of tweets. From each subset, $30 \%$ was set aside to make up the test set for validation. Table 3 shows the distribution of tweets for the six emotion classes in the training and test datasets that were used in the experiments.

Table 3. Number of tweets in the training and test datasets

\begin{tabular}{|l|c|c|}
\hline Emotion & Training & Test \\
\hline anger & 13,226 & 5,669 \\
\hline fear & 13,226 & 5,669 \\
\hline happy & 13,226 & 5,669 \\
\hline love & 13,226 & 5,669 \\
\hline sadness & 13,226 & 5,669 \\
\hline surprise & 13,226 & 5,669 \\
\hline Total Tweets & $\mathbf{7 9 , 3 5 6}$ & $\mathbf{3 4 , 0 1 4}$ \\
\hline
\end{tabular}

\subsection{Experimental Settings}

The experiments were conducted on the code-mixed dataset using SVM as the base learner, as described in Section 3. The initial labeled dataset was created by randomly extracting 100 tweets from each emotion class of the training set, thus yielding a total of 600 tweets which were than shuffled to ensure a randomized distribution of emotion labels within the initial labeled dataset. To create the unlabeled dataset, the emotion labels of the remaining 78,756 tweets in the training set were removed and then shuffling was performed.

A total of 10 iterations were carried out. At the end of each iteration, the data with predicted labels that had confidence scores that met the threshold were removed from the unlabeled dataset and added to the labeled dataset to create a new training set for the next iteration. In the experiments, the following aspects of self-training were explored.

- Self-training with different confidence thresholds. To study the effect of using different confidence thresholds, different threshold values ranging from 0.65 to 1.00 with increments of 0.05 (i.e., $0.65,0.70$, 0.75 , etc.) were used. The initial labeled dataset comprised of 600 tweets. The features comprised of unigrams with TF-IDF set to a max_df of 0.7 , which meant that $70 \%$ of the most frequently used terms were excluded as these terms appeared with high frequency among the tweets in the training set and were therefore not able to provide much discriminative value of the different emotions.

- Self-training with different n-gram feature sets. Experiments using three different features sets unigrams, bigrams and trigrams - with TF-IDF were carried out. The initial labeled dataset consisted of 600 tweets and the confidence threshold was set at 0.80 .

\section{RESULTS}

The performances of the models were evaluated using the balanced hold-out set comprising a total of 34,014 tweets as shown in Table 3. The results of the self-training approach with different confidence thresholds are 
shown in Table 4 and Figure 1. The accuracy of the self-trained classifier reached a plateau after the second or third iterations. This was consistent with the growth in the labeled dataset size depicted in Figure 2 which increased greatly in the second or third iterations before reaching a plateau as well. The accuracy achieved when using lower confidence thresholds were much lower compared to higher thresholds as adding labeled data with lower prediction confidence scores to the training set for the subsequent iterations would result in lower accuracy (Iosifidis and Ntoutsi, 2019). However, the final accuracies obtained by self-training with the various thresholds were still higher than the baseline SVM model which achieved an accuracy of $84 \%$.

The ability to enlarge the labeled training set by adding data with high confidence predicted labels throughout the iterations is important as a means to supplement the limited labeled data, considering that it is very expensive to produce manually labeled datasets. Moreover, to ensure good accuracy when predicting the emotion labels of new (previously unseen) tweets, there is a need for classifiers to be trained using a substantially large dataset (Mishne, 2005).

Table 4. Accuracy per iteration for self-training with various thresholds

\begin{tabular}{|c|c|c|c|c|c|c|c|c|c|c|}
\hline & \multicolumn{9}{|c|}{ Iterations } \\
\hline Threshold & $\mathbf{1}$ & $\mathbf{2}$ & $\mathbf{3}$ & $\mathbf{4}$ & $\mathbf{5}$ & $\mathbf{6}$ & $\mathbf{7}$ & $\mathbf{8}$ & $\mathbf{9}$ & $\mathbf{1 0}$ \\
\hline $\mathbf{0 . 6 5}$ & 0.84 & 0.87 & 0.87 & 0.87 & 0.87 & 0.87 & 0.87 & 0.87 & 0.87 & 0.87 \\
\hline $\mathbf{0 . 7 0}$ & 0.84 & 0.87 & 0.88 & 0.88 & 0.88 & 0.88 & 0.88 & 0.88 & 0.88 & 0.88 \\
\hline $\mathbf{0 . 7 5}$ & 0.84 & 0.87 & 0.88 & 0.88 & 0.88 & 0.88 & 0.88 & 0.88 & 0.88 & 0.88 \\
\hline $\mathbf{0 . 8 0}$ & 0.84 & 0.87 & 0.88 & 0.88 & 0.89 & 0.89 & 0.89 & 0.89 & 0.89 & 0.89 \\
\hline $\mathbf{0 . 8 5}$ & 0.84 & 0.87 & 0.87 & 0.88 & 0.88 & 0.88 & 0.88 & 0.88 & 0.88 & 0.88 \\
\hline $\mathbf{0 . 9 0}$ & 0.84 & 0.87 & 0.88 & 0.88 & 0.89 & 0.89 & 0.89 & 0.89 & 0.89 & 0.89 \\
\hline $\mathbf{0 . 9 5}$ & 0.84 & 0.86 & 0.87 & 0.87 & 0.88 & 0.88 & 0.88 & 0.88 & 0.88 & 0.89 \\
\hline
\end{tabular}

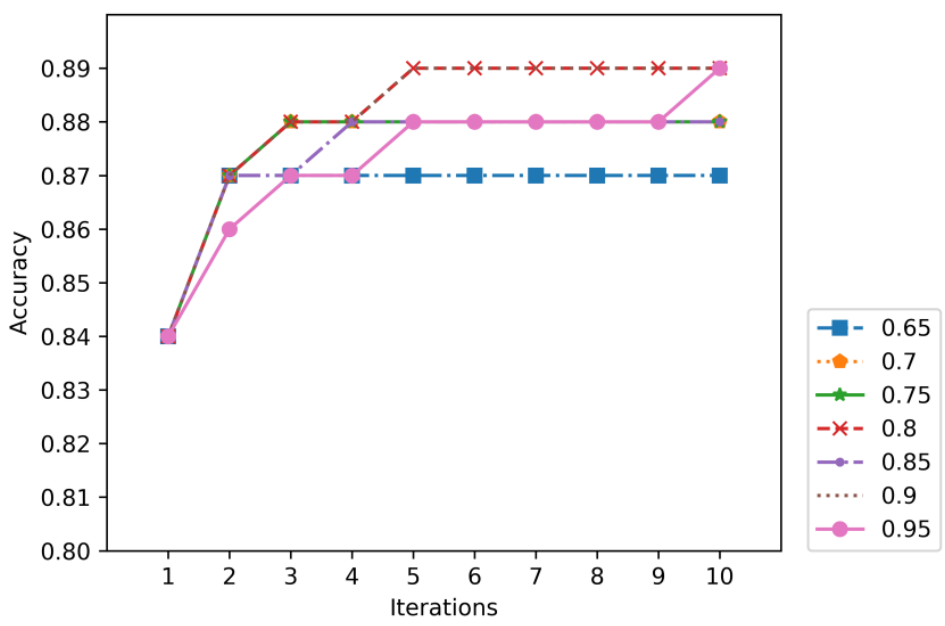

Figure 1. Accuracy of self-training with unigram features and different confidence thresholds 


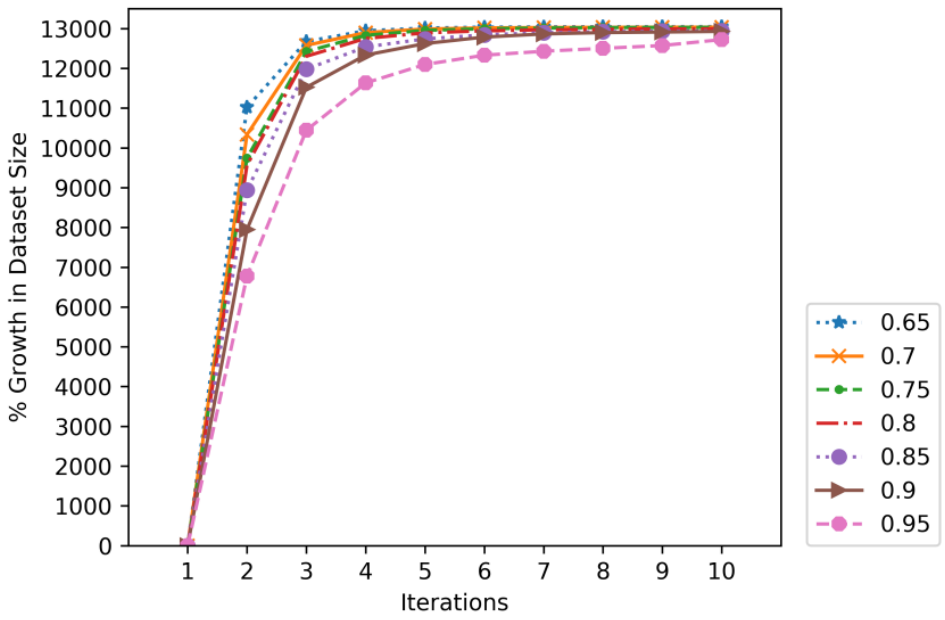

Figure 2. Labeled dataset growth of self-training with unigram features and different confidence thresholds

Self-training with n-gram features also outperformed the baseline SVM models, as shown in Figure 3. Among the n-gram features, unigrams provided the best performance compared to bigrams and trigrams. As the predicted labels are able to achieve confidence scores exceeding the threshold of 0.8 , the accuracy obtained are higher compared to that of the baseline model. This is promising as it shows the potential of using self-training in situations where there is limited labeled code-mixed microblog datasets available for training. It was observed that the accuracy achieved by using unigram features achieved a higher accuracy compared to bigram and trigram. This is most likely due to the fact that for $n>1$, the constituent individual terms in the $n$-gram would appear as separate parallel features together with the $n$-gram itself in the feature vector resulting in some duplication of features (Tang et al., 2015). For example, if the trigram "best day ever" appeared in a tweet, the list of features for the tweet would include the terms "best day ever", "best day", "day ever", "best", "day" and "ever". To address this problem, the n-grams have to be filtered such that only multiword expressions (MWE) would be retained and their constituent terms are removed from the feature vector of the tweet concerned. However, this would require the availability of an appropriate code-mixed MWE lexicon.

All three n-gram features used were able to expand the labeled dataset and had the largest growth rate during the second and third iterations as shown in Figure 4.

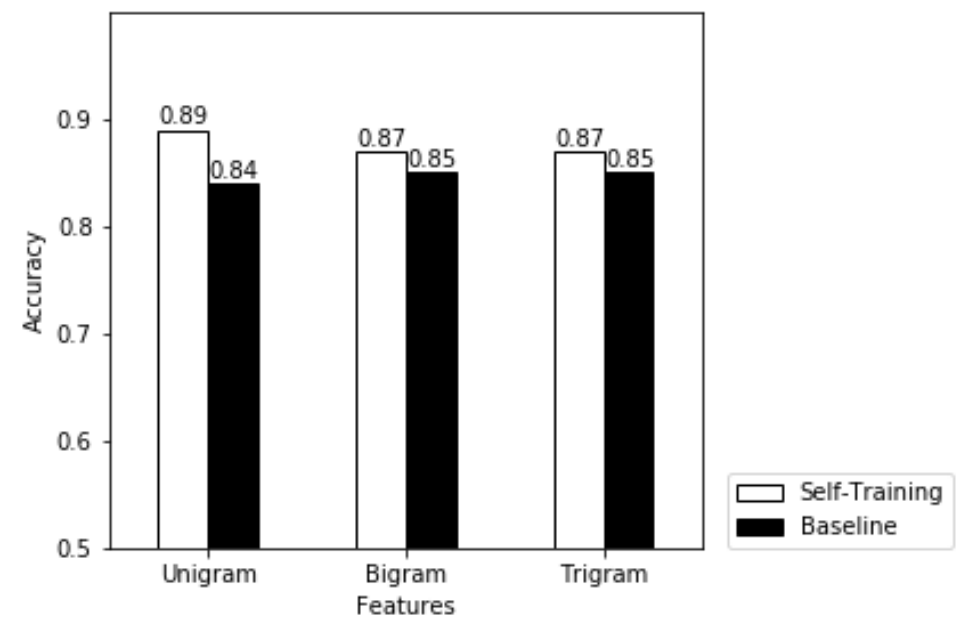

Figure 3. Accuracy of self-training with various n-gram features 


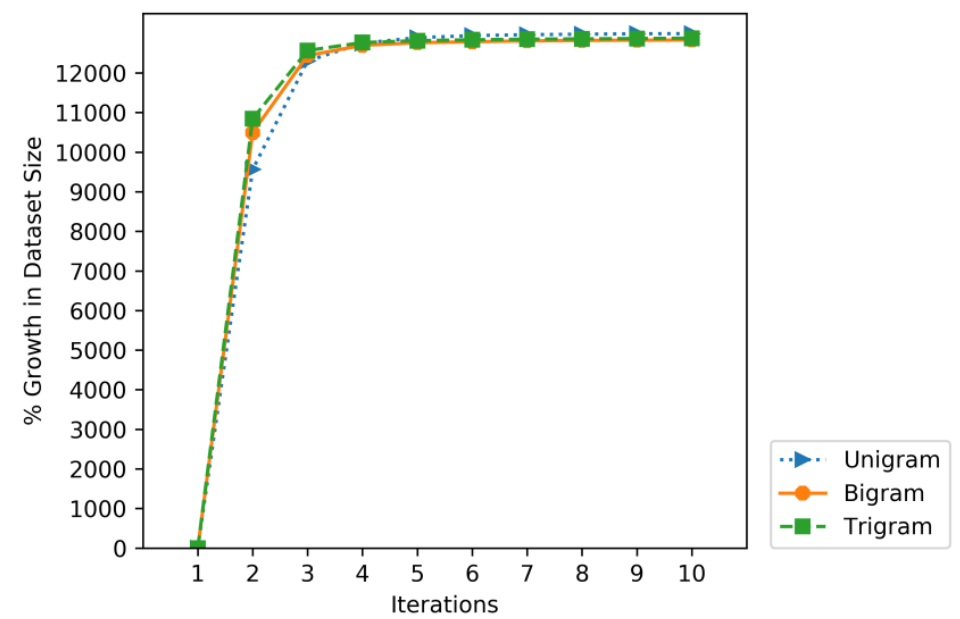

Figure 4. Labeled set growth per iteration for self-training with various n-gram features

The comparison of individual F1-scores for each emotion in Table 5 showed that the self-training classifiers were able to achieve a better performance compared to the baseline models. The emotion labels that achieved the best results with F1-scores above 0.90 were anger, fear and happy. This is probably attributed to the consistent way in which people expressed these emotions in the tweets that they write. On the other hand, the emotion that obtained the lowest F1-score was sadness. This could be due to either the implicit expression of this emotion and the use of sarcasm or irony which is difficult to detect in tweets.

Table 5. F1-score by emotion label for various n-gram features

\begin{tabular}{|c|c|c|c|c|c|c|}
\hline n-gram & \multicolumn{2}{|c|}{ unigram } & \multicolumn{2}{c|}{ bigram } & \multicolumn{2}{c|}{ trigram } \\
\hline Emotion & baseline & self-training & baseline & self-training & baseline & self-training \\
\hline anger & 0.90 & $\mathbf{0 . 9 4}$ & $\mathbf{0 . 9 1}$ & $\mathbf{0 . 9 1}$ & 0.91 & $\mathbf{0 . 9 2}$ \\
\hline fear & 0.95 & $\mathbf{0 . 9 8}$ & 0.94 & $\mathbf{0 . 9 7}$ & 0.94 & $\mathbf{0 . 9 5}$ \\
\hline happy & 0.92 & $\mathbf{0 . 9 3}$ & 0.92 & $\mathbf{0 . 9 3}$ & 0.92 & $\mathbf{0 . 9 3}$ \\
\hline love & 0.84 & $\mathbf{0 . 9 1}$ & 0.84 & $\mathbf{0 . 8 6}$ & 0.84 & $\mathbf{0 . 8 5}$ \\
\hline sadness & 0.70 & $\mathbf{0 . 7 5}$ & 0.71 & $\mathbf{0 . 7 5}$ & 0.71 & $\mathbf{0 . 7 5}$ \\
\hline surprise & 0.75 & $\mathbf{0 . 8 0}$ & 0.76 & $\mathbf{0 . 8 0}$ & 0.76 & $\mathbf{0 . 8 1}$ \\
\hline average & 0.84 & $\mathbf{0 . 8 9}$ & 0.85 & $\mathbf{0 . 8 7}$ & 0.85 & $\mathbf{0 . 8 7}$ \\
\hline
\end{tabular}

\section{CONCLUSION}

Code-mixed microblog text presents challenges to emotion analysis due to its use of informal language and mixture of languages, as well as its lack of proper structure. In this paper, self-training with SVM was used to carry out emotion analysis of Malaysian code-mixed Twitter data. The experiments carried out evaluated the use of different confidence thresholds as well as various n-gram features. The results are promising as a relatively high accuracy was achieved even with a small initial labeled dataset, which is ideal for situations where labeled datasets for emotion analysis is very limited.

For future work, the use of other classifiers as the base learners should be investigated. There is also a need to explore the effect of using other type of features including syntactic language features such as part-of-speech (POS), emoticons, and emotion-bearing terms. In addition, the importance of ensuring that the resulting enlarged labeled dataset generated at the end of each iteration has a balanced representation of each emotion should also be studied. 


\section{REFERENCES}

Aghababaei, S. and Makrehchi, M., 2017. Interpolative self-training approach for sentiment analysis. IEEE/ACM BESC 2016 - Proceedings of 2016 International Conference on Behavioral, Economic, Socio - Cultural Computing, pp.1-6.

Blitzer, J., Dredze, M. and Pereira, F., 2007. Biographies, bollywood, boom-boxes and blenders: Domain adaptation for sentiment classification. ACL 2007 - Proceedings of the 45th Annual Meeting of the Association for Computational Linguistics, pp.440-447.

Crammer, K., Kulesza, A. and Dredze, M., 2013. Adaptive regularization of weight vectors. Machine Learning, 91(2), pp.155-187.

Dashtipour, K. et al., 2016. Multilingual Sentiment Analysis: State of the Art and Independent Comparison of Techniques. Cognitive Computation, 8(4), pp.757-771.

Haimovitch, Y., Crammer, K. and Mannor, S., 2012. More is better: Large scale partially-supervised sentiment classification. Journal of Machine Learning Research, 25, pp.175-190.

Han, Y., Liu, Y. and Jin, Z., 2019. Sentiment analysis via semi-supervised learning: a model based on dynamic threshold and multi-classifiers. Neural Computing and Applications, 32(9), pp.5117-5129. Available at: https://doi.org/10.1007/s00521-018-3958-3.

He, Y. and Zhou, D., 2011. Self-training from labeled features for sentiment analysis. Information Processing and Management, 47(4), pp.606-616. Available at: http://dx.doi.org/10.1016/j.ipm.2010.11.003.

Hemmatian, F. and Sohrabi, M.K., 2019. A survey on classification techniques for opinion mining and sentiment analysis. Artificial Intelligence Review, 52(3), pp.1495-1545. Available at: https://doi.org/10.1007/s10462-017-95996.

Husein, Z., 2018. Malay-Dataset. Available at: https://github.com/huseinzol05/Malay-Dataset.

Iosifidis, V. and Ntoutsi, E., 2019. Sentiment analysis on big sparse data streams with limited labels. Knowledge and Information Systems. Available at: https://doi.org/10.1007/s10115-019-01392-9.

Lo, S.L., Cambria, E., Chiong, R. and Cornforth, D., 2016. A multilingual semi-supervised approach in deriving Singlish sentic patterns for polarity detection. Knowledge-Based Systems, 105, pp.236-247.

Mann, G.S. and McCallum, A., 2010. Generalized expectation criteria for semi-supervised learning with weakly labeled data. Journal of Machine Learning Research, 11, pp.955-984.

Mishne, G., 2005. Experiments with mood classification in blog posts. Proceedings of ACM SIGIR 2005 workshop on stylistic .... Available at: http://citeseerx.ist.psu.edu/viewdoc/download?doi=10.1.1.111.2693\&rep=rep1\&type=pdf.

Pang, B. and Lee, L., 2005. Seeing Stars: Exploiting class relationships for sentiment categorization with respect to rating scales. Proceedings of ACL 2005. 2005 pp. 33-40.

Da Silva, N.F.F., Coletta, L.F.S. and Hruschka, E.R., 2016. A survey and comparative study of tweet sentiment analysis via semi-supervised learning. ACM Computing Surveys, 49(1), pp.1-26.

Statcounter, 2020. Social Media Stats Malaysia - April 2019 - April 2020. Available at: https://gs.statcounter.com/socialmedia-stats/all/malaysia.

Tang, D. et al., 2015. A Joint Segmentation and Classification Framework for Sentence Level Sentiment Classification. IEEE Transactions on Audio, Speech and Language Processing, 23(11), pp.1750-1761.

The Proteus Project, 2004. The Proteus Project. Available at: https://nlp.cs.nyu.edu/GMA_files/resources/malay.stoplist.

Wilson, T., Wiebe, J. and Hoffmann, P., 2005. Recognizing contextual polarity in phrase-level sentiment analysis. HLT/EMNLP 2005 - Human Language Technology Conference and Conference on Empirical Methods in Natural Language Processing, Proceedings of the Conference, pp.347-354. 